und so weiter. Mathematisch sieht dies so aus:

$$
e=e_{0}\left(1-A+A^{2}-A^{3}+\ldots\right)=\frac{e_{0}}{1+A},
$$

wobei $A$ eine unendlich große Konstante ist. Sie läßt sich leicht in erster Näherung berechnen und führt dann bekanntlich auf ein divergentes Integral. Dies hat zur Folge, daß jede endliche Ladung ausgelöscht wird. Es ist nun die Frage, ob nicht durch die höheren Näherungen, also durch die Wechselwirkung der Paare mit dem Strahlungsfeld, die Ansammlung von unendlich vielen Paaren verhindert wird und sich so ein Gleichgewicht einspielt, ohne daß die Ladung ganz vernichtet wird. Um diese Frage zu beantworten, müßte man die entsprechenden Elemente der S-Matrix exakt ausrechnen, was noch niemand gelungen ist. Es soll hier nur kurz gezeigt werden, daß die üblichen Argumente, nach welchen die höheren Näherungen genau so divergieren wie die erste, nicht unbedingt richtig sind.

Den strengen Ausdruck für die Vakuumpolarisation erhält man nach der bekannten D y s o n schen Vorschrift dadurch, daß man in dem irreduziblen Graph einen Eckpunkt durch den strengen Scheitelanteil und die Fortpflanzungsfunktion für die Elektronen $S$ durch die strenge Funktion $S^{\prime}$ ersetzt. Für letztere erhält man im Impulsraum folgenden Ausdruck:

$$
S_{(p)}^{\prime}=\frac{S_{(p)}}{1-S_{(p)} \Sigma_{(p)}} .
$$

$\Sigma$ ist der Beitrag von unzerlegbaren Selbstenergie-Graphen, über alle zerlegbaren Graphen ist bereits summiert. In Potenzen von $\alpha$ entwickelt, berechnen sich die ersten Terme von $\Sigma$ zu

$$
\begin{aligned}
& \sum_{(p)}^{1}=\frac{a}{4 \pi} S_{(p)}^{-1} \ln a \\
& \sum_{(p)}^{2}=-\left(c \frac{a}{4 \pi}\right)^{2} S_{(p)}^{-1}(\ln a)^{2}
\end{aligned} \quad a=\frac{p^{2}-m^{2}}{m^{2}} .
$$

$p$ ist der Energie-Impuls Vektor des Elektrons, $c$ eine Konstante der Ordnung 1. Diese Formeln gelten nur für den Grenzfall $a \gg 1$.

Was Beiträge mit höheren Potenzen von $\alpha$ anlangt, so kann man allgemein zeigen, daß sie nicht stärker als eine Potenz von $\ln a$ anwachsen und daß das Glied mit der $n$-ten Potenz von $\alpha$ auch die $n$-te Potenz von $\ln a$ enthält. Den noch unbestimmten numerischen Koeffizienten für das allgemeine Glied zu finden, ist mir nicht gelungen.

Es herrscht nun allgemein die Ansicht, daß das Einsetzen von $S^{\prime}$ an die Stelle von $S$ nichts an der Konvergenz eines Integrals ändert, da sie sich ja nur um logarithmische Faktoren unterscheiden. Aber gerade die schwachen, logarithmischen Divergenzen, welche in der Quantenelektrodynamik auftreten, können durch solche Faktoren beseitigt werden. Begnügt man sich etwa mit obigen Korrektionstermen und approximiert die strenge S-Funktion durch $S^{\prime}=S(1+c \alpha \ln a)^{-2}$, dann erhält man für die Selbstladung folgenden Ausdruck:

$$
\int \frac{\mathrm{d} p}{\left(p^{2}-m^{2}\right)\left[1+c a \ln \left(\left(p^{2}-m^{2}\right) / m^{2}\right)\right]^{2}\left[(p-k)^{2}-m^{2}\right]\left[1+c c \ln \left((p-k)^{2}-m^{2}\right) / m^{2}\right]^{2}}
$$

Er ist für den Limes verschwindenden Fourier-Vektors $k$ des elektrischen Feldes zu berechnen. Dieser Ausdruck stellt also die Summe aller Photon-Selbstenergie-Graphen dar, die zerlegbare Elektron-Selbstenergie-Graphen enthalten, welche sich auf unzerlegbare zweiter oder vierter Ordnung zurückführen lassen. Für Impulse größer als $P$ mit $c \alpha \ln P k=1$ werden die logarithmischen Korrektionsglieder bemerkbar und machen das Integral konvergent. Es berechnet sich für hohe Impulse zu

oder mit

$$
\int_{P}^{\infty} \frac{\mathrm{d}|p|}{|p|(c a \ln |p| k)^{2}}
$$

$$
u=\ln |p| k ; \quad c^{-2} \alpha^{-2} \int_{1}^{\infty} c \alpha \frac{\mathrm{d} u}{u^{2}}=1 / c \alpha .
$$

Wenn man im Integral $(1+c \alpha \ln )^{-2}$ nach Potenzen von $\alpha$ entwickelt: $1-2 c \alpha \ln +\ldots$, dann divergiert das Integral über jedes Glied. Das ist vernünftig, denn $\alpha^{-1}$ läßt sich nicht nach Potenzen von $\alpha$ entwickeln. Für die Selbstladung würde man in dieser Näherung

$e=e_{0}(1+\alpha \cdot 1 / c \alpha)$ cder $e / e_{0}=1+1 / c$ erhalten.

Zusammenfassend können wir also sagen, daß in einer strengen Theorie wahrscheinlich keine Unendlichkeiten auftreten werden. Es scheint ein glücklicher Zufall zu sein, daß man in der Quantenelektrocynamik nach $\mathrm{Re}$ - normalisation die beobachtbaren Größen nach Potenzen von $\alpha$ entwickeln kann, während die Renormalisationsterme selbst sich nicht nach Potenzen von $\alpha$ entwickeln lassen. Die allerdings rein akademische Frage, ob letztere endlich oder unendlich sind, wird sich erst beantworten lassen, wenn eine Lösung ohne Reihenentwicklung nach $\alpha$ vorliegt.

\section{Beobachtung von Ionen-Dissoziationen mit Hilfe eines Parabelspektrographen}

Von H. Ewald und A. Henglein Max-Planck-Institut für Chemie, Mainz

(Z. Naturforschg. 6 a, 463-464 [1951]; eingeg. am 18. Juni 1951)

Für massenspektrographische Dublettmessungen wurde eine Elektronenstoß-Ionenquelle nach $\mathrm{S} \mathrm{haw}{ }^{\mathbf{1}}$ mit Hilfe eines Thomsonschen Parabelspektrographen entwickelt. Eine solche Quelle liefert im Gegensatz zur Kanalstrahlröhre Ionen sehr homogener Energie. Dementsprechend markieren sich die von der Quelle gelieferten Ionen verschiedener Massen auf dem Leuchtschirm bzw. der Photoplatte des Parabelspektrographen nicht als ausgedehnte Parabelstücke, sondern nur als definierte Punkte derselben, die gleicher Energieablenkung der Strahlen durch das elektrische Feld entsprechen. Diese Punkte (Hauptpunkte)

1 A. E. S h aw , Physic. Rev. 75, 1011 [1949]. 
werden auf den Aufnahmen in großer Intensität beobachtet (in Abb. 1 bei der Abszisse $x_{0}$ ).

Bei erhöhtem Druck in der Ionenquelle und dementsprechend auch in der übrigen Apparatur erhalten diese Punkte aber schwächere Fortsätze in Richtung der Parabeln. Das bedeutet, daß dann neben der Elektronenstoß-Ionisation im Stoßraum der Ionenquelle eine zusätzliche Ionenbildung durch sekundäre Prozesse auf der Beschleunigungsstrecke der Ionen einsetzt. Die so gebildeten Ionen haben ge-

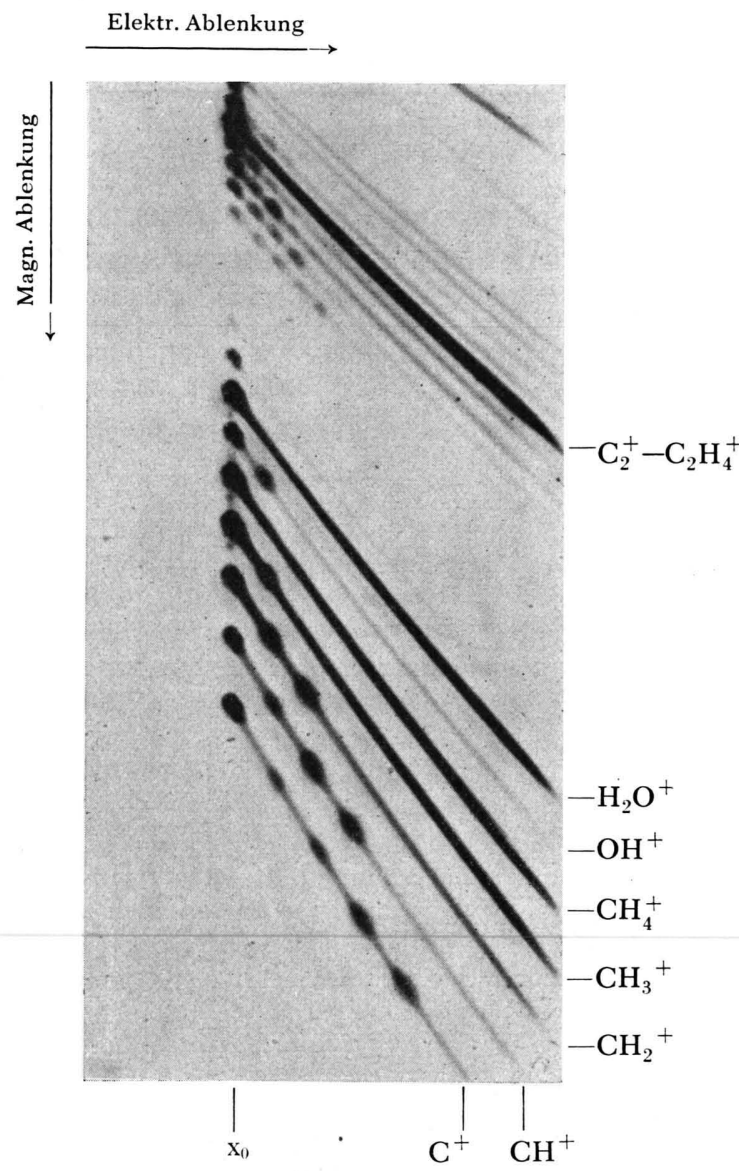

Abb. 1. Dissoziationsspektrum der Bestandteile des Leuchtgases.

ringere Energie und kommen daher zu Parabelpunkten, die größerer Ablenkung in den Feldern entsprechen.

Nun werden aber auf den schwächeren Parabelfortsätzen in größerer Anzahl auch Nebenpunkte erheblicher Intensität beobachtet. Diese sind zurückzuführen auf Dissoziationen, die die primär im Stoßraum der Quelle gebildeten Ionen im feldfreien Raum zwischen der Beschleunigungsstrecke und den Ablenkfeldern durch $\mathrm{Zu}$ sammenstöße mit Restgasmolekülen erfahren. Die so sekundär entstehenden Ionen haben eine kleinere Energie als die primär im Stoßraum gebildeten, da sich deren kinetische Energie im Verhältnis der Massen auf die Bruchstücke aufteilt. Mehr oder weniger ungleichmäßige Intensitätsverteilungen längs der Parabeln, hervorgerufen durch solche langsamen Ionen, sind auch schon früher bei Betrieb mit Kanalstrahlröhren auf den Spektrogrammen aufgetreten und als Folge von Dissoziationen gedeutet wcrden 2 .

Diese Stoßdissoziationen werden auch in doppelfokussierenden Massenspektrographen häufig beobachtet und sind darin besonders eingehend von $\mathrm{Mat}$ a $\mathrm{uch}$ und L i c h t b l a u ${ }^{3}$ untersucht worden. Die ionisierten Bruchstücke der Dissoziationen erscheinen in den Spektrogrammen clieser Apparate als diffuse Linien (Banden), die auch bei nicht-ganzzahligen Massenzahlen liegen können.

Die Ergebnisse von $\mathrm{Mattauch}$ und $\mathrm{Lichtblau}$ können an Hand der jetzt mit dem Parabelspektrographen erhaltenen Aufnahmen in vieler Beziehung bestätigt und auch erweitert werden. Dabei erweisen sich die Parabelspektrogramme als geeigneter für solche Untersuchungen, da aus der Lage der Dissoziationspunkte direkt die wahre Massenzahl und die Energie der sekundären Ionen entnommen werden kann.

Jeder einheitlichen Verbindung kommt ein charakteristisches Dissoziationsspektrum zu. Abb. 1 zeigt als Beispiel einen Ausschnitt aus dem für Leuchtgas erhaltenen Parabelspektrogramm. Die Parabeln der Massenzahlen 12, 13, 14 und 15 weisen neben den Hauptpunkten der Energieablenkung $\mathrm{x}_{0}$, die den Primärionen $\mathrm{C}^{+}, \mathrm{CH}^{+}, \mathrm{CH}_{2}^{+}$bzw. $\mathrm{CH}_{3}^{+}$. entsprechen, 4, 3, 2 bzw. einen Nebenpunkt auf. Diese Punkte stellen einen Teil des Dissoziationsspektrums des im Leuchtgas enthaltenen Methans dar. Die C+-Ionen z. B., die den vier Nebenpunkten der C+-Parabel entsprechen, entstehen durch Dissoziation aus den primären $\mathrm{CH}^{+}-, \mathrm{CH}_{2}^{+}-\mathrm{CH}_{3}^{+}$- und $\mathrm{CH}_{4}^{+}$-Ionen. Der Nebenpunkt auf der Parabel der Massenzahl 17 ist zurückzuführen auf die Bildung von $\mathrm{OH}^{+}$-Ionen aus primären $\mathrm{H}_{2} \mathrm{O}+$-Teilchen. Bei den Massenzahlen 24-28 sind die Dissoziationspunkte des Äthylens erkennbar.

2 s. W. W i e n, Hdb. d. Exp.-Physik XIV, 1927.

3 J. Mat ta u ch u. H. Li chtblau, Physik. Z. 40, 16 [1939].

\section{Komplementäre Filtermethode für Raman-Untersuchungen an Kristallpulvern}

Von W. Gu ber und K. H. Rig ger t

I. Physikalisches Institut der Universität Heidelberg

(Z. Naturforschg. 6 a, 464-466 [1951]; eingeg. am 25. Juni 1951)

Bei Ausdehnung der Raman-Untersuchungen von Flüssigkeiten auf Kristallpulver zeigt.sich infolge der Reflexion an den einzelnen Kristalloberflächen eine starke Überbelichtung der Erregerlinie und des unmittelbar benachbarten Spektralbereiches, was zu einer Verschleierung der naheliegenden Raman-Linien führt. Um dies zu beheben, benötigt man eine ideale komplementäre Filteranorchung. $\mathrm{Zu}$ deren Verwirklichung sind bisher nur wenige erfolgreiche, oft sehr komplizierte Methoden ausgearbeitet worden ${ }^{1-5}$. Einer von uns $^{5}$ wies in einer früheren Arbeit

1 W. G e r la c h, Ann. Physik 5, 196 [1930]. (Immersionsmethode und Methode der gekreuzten Filter.)

2 J. H i b b e n, Chem. Rev. 13, 345 [1933]. (Immersionsmethode.) 